Nima H. Siboni*, Gaurav P. Shrivastav, Stavros D. Peroukidis and Sabine H. L. Klapp

\title{
Structure and rheology of soft hybrid systems of magnetic nanoparticles in liquid-crystalline matrices: results from particle-resolved computer simulations
}

\begin{abstract}
Hybrid mixtures composed of magnetic nanoparticles (MNP) in liquid crystalline (LC) matrices are a fascinating class of soft materials with intriguing physical properties and a wide range of potential applications, e.g., as stimuliresponsive and adaptive materials. Already in the absence of an external stimulus, these systems can display various types of orientationally disordered and ordered phases, which are enriched by self-assembled structures formed by the MNPs. In the presence of external fields, one typically observes highly nonlinear macroscopic behavior. However, an understanding of the structure and dynamics of such systems on the particle level has, so far, remained elusive. In the present paper we review recent computer simulation studies targeting the structure, equilibrium dynamics and rheology of LC-MNP systems, in which the particle sizes of the two components are comparable. As a numerically tractable model system we consider mixtures of soft spherical or elongated particles with a permanent magnetic dipole moment and ellipsoidal non-magnetic particles interacting via a Gay-Berne potential. We address, first, equilibrium aspects such as structural organization and self-assembly (cluster formation) of the MNPs in dependence of the orientational state of the matrix, the role of the size ratio, the impact of an external magnetic field, and the translational and orientational diffusion of the two components. Second, we discuss the non-equilibrium dynamics of LC-MNP mixtures under planar shear flow, considering both, spherical and non-spherical MNPs. Our results contribute to a detailed understanding of these intriguing hybrid materials, and they may serve as a guide for future experiments.
\end{abstract}

\footnotetext{
*Corresponding author: Nima H. Siboni, Institut für Theoretische Physik, Technische Universität Berlin, Hardenbergstraße 36, 10623 Berlin, Germany, e-mail: hamidisiboni@tu-berlin.de. https://orcid.org/ 0000-0002-0305-5870
}

Gaurav P. Shrivastav, Institute für Theoretical Physics, Technische Universität Wien, Wiedner Hauptstr. 8-10, 1040 Vienna, Austria

Stavros D. Peroukidis, Department of Chemical Engineering, University of Patras, 26504 Patras, Greece; and Hellenic Open University, 26222, Patras, Greece

Sabine H. L. Klapp, Institut für Theoretische Physik, Technische Universität Berlin, Hardenbergstraße 36, 10623 Berlin, Germany 
Keywords: magnetic liquid crystal mixtures, molecular dynamics simulations, Monte Carlo simulations, rheology, structure

\section{Introduction}

Hybrid mixtures composed of magnetic nanoparticles (MNP) in liquid crystalline (LC) matrices have attracted attention about five decades: Already in 1970, Brochard and de Gennes predicted theoretically intriguing features of such "ferronematic" systems [1], including spontaneous magnetization and giant field induced effects. In fact, LC-MNP mixtures combine, in a synergistic way, various features of their constituents which are highly attractive already on their own: LCs, that is, molecular or colloidal systems of anisometric particles, show various ordered (liquid-crystalline) phases as well as phase separation, electric-optical switching behavior in external fields, and a nonlinear rheological behavior under shear, to name just a few examples. Suspensions of MNPs (or larger magnetic particles) show superparamagnetic behavior (see Refs. [2, 3] for reviews), giving rise to a wide range of applications. More fundamentally, magnetic particles are prototypes of self-assembling systems forming clusters, chains and other structures due to strong dipole-dipole interactions. This short list already suggests highly complex structural and dynamical behavior of LC-MNP mixtures.

The marked field sensitivity predicted by Brochard and de Gennes arises even for small concentrations of (spherical) MNPs and has been studied in many experiments involving different LC-MNP mixtures [4-10], including systems on the colloidal scale [11, 12]. Recently, new interest in LC-MNP mixtures was stimulated by the discovery $[13,14]$ of spontaneous magnetic ordering in a hybrid system of large, micron-sized magnetic plates embedded in a thermotropic LC. A crucial ingredient for the observed behavior are the strong local distortions of the LC director induced by the magnetic plates (whose size is much larger than that of the LCs). These distortions yield, in turn, pronounced anisotropic, effective (i.e., LC-mediated, elastic) interactions. Today, magnetic particles can be fabricated in a range of different sizes and in different shapes [5, 8, 9, 13, 15-18], and one would expect that these geometrical properties play a key role for the complex behavior of the mixture as a whole. On the one hand, the particle shape will influence the distortions and thus, the LC-mediated elastic interactions [19-24] between the MNPs. On the other hand, the size of the magnetic particles (affecting their magnetic moment) and their shape determine the self-assembly behavior, which crucially depends on the (shape-dependent) lowest-energy configuration of a pair of particles. For example, while two spherical MNPs arrange in a head-to-tail configuration, this is not the case for e.g., ellipsoidal MNPs [25, 26].

Despite the strong experimental interest in LC-MNP mixtures and the significant developments in the design of different composites from a chemical point of view, the microscopic (i.e., particle-resolved) structure of such systems is, in many cases, essentially unexplored. From the theoretical perspective, ferronematics involving large MNPs have mainly been studied on the basis of Landau-de Gennes free energy 
approaches [7, 27, 28], focusing on the impact of elastic distortions. While this continuum-type of approach is quite successful to explain, e.g., the spontaneous magnetization of the platelet system in Refs. [13, 14], it does not reveal particle-resolved information. This seems particularly important when it comes to mixtures in which the sizes of magnetic particles and LCs are comparable, such as lyotropic suspensions of colloid pigment rods and magnetite particles [11, 12]. The most appropriate theoretical method to study such systems on a particle level is many-particle computers simulations. However, until recently this type of method was not applied to LC-MNP mixtures, presumably due to the large computational effort required to handle the involved interactions which act on many length scales.

Driven by this lack, we have recently started a number of computer simulation studies targeting the structure, equilibrium dynamics and rheology of LC-MNP systems, in which the particle sizes of the two components are of the same order of magnitude [12, 29-34]. The present review summarizes main results and points out open questions. As a numerically tractable model system we consider mixtures of soft, spherical or elongated particles with an embedded permanent magnetic dipole moment and ellipsoidal non-magnetic particles interacting via a Gay-Berne (GB) potential. To analyze the resulting collective behavior, we have employed Monte Carlo (MC) and Molecular Dynamics (MD) simulations in equilibrium and under shear flow.

The article is organized as follows Section 2 is devoted to the equilibrium structure of mixtures involving spherical MNPs with different sizes relative to that of the LC particles. We review the phase behavior, cluster formation, and the impact of a constant magnetic field. In Section 3 we discuss the equilibrium dynamics in LC-MNP mixtures, particularly the translational motion in the zero-field case. In Section 4 we then turn to the non-equilibrium dynamic of mixtures driven by external shear flow. This is a particularly interesting issue in the context of rheology (for reviews of the shear-induced behavior of the pure components, such as the shear-induced ordering in pure LC systems or the magnetoviscous effects in systems solely composed of the MNPs, see [35-37].) Finally, we provide a brief outlook in Section 5.

\section{Structure of LC-MNP mixtures}

In this section we discuss the structure of mixtures of spherical MNPs and LCs in thermal equilibrium. We start by introducing our model systems and then summarize recent computer simulation studies based on the MC and equilibrium MD simulations [12, 29-31, 38].

\subsection{Model and simulations details}

Within our particle-resolved simulations the nonmagnetic LC component is represented by a GB rod and the MNP is modeled as a dipolar soft sphere (DSS). The latter has 
an embedded, permanent point dipolar moment of strength $\mu$ in the center. The GB rods are characterized by a length $(l)$ to width $(\sigma)$ ratio set to $l / \sigma=3$. Pure GB systems [39] with this aspect ratio are very well studied and can serve as a reference. The DSS have a diameter $\sigma_{\mathrm{s}}$. The components of the mixtures have sizes of the same order of magnitude, with $\sigma_{\mathrm{s}}^{\star}=\sigma_{\mathrm{s}} / \sigma$ ranging from one fourth of the width of the GB rods, i.e., $\sigma_{\mathrm{s}}^{\star}=1 / 4$ (in this case we refer to "small” MNPs), to two times, i.e., $\sigma_{\mathrm{s}}^{\star}=2$. We note that with much smaller or larger values of $\sigma_{\mathrm{s}}^{\star}=\sigma_{\mathrm{s}} / \sigma$, the simulations tend to become unrealistic. The spheres interact via a soft repulsive potential and a long range dipole-dipole potential. Long-range interactions are treated with the three-dimensional Ewald sum [40]. To measure the strength of the interactions we define the reduced dipole moment $\mu^{\star}=\mu / \sqrt{\epsilon_{0} \sigma_{\mathrm{s}}^{3}}$ and the reduced temperatures $T^{\star}=k_{\mathrm{B}} T / \epsilon_{\mathrm{O}}$ (where $k_{\mathrm{B}}$ is the Boltzmann constant and $\epsilon_{0}$ is the soft-sphere energy parameter). Further we use the dipolar coupling parameter $\lambda=\mu^{2} / k_{B} T \sigma_{\mathrm{s}}^{3}$ that takes values between $\approx 3.5$ and $\approx 15$, consistent with values characterizing real, strongly coupled MNPs [41-43]. Finally, a modified GB potential [30] has been used for the interaction between rods and spheres that depends only on the orientation of the rods and the inter-particle distance vector of the center of mass (CoM) of rods and spheres.

\subsection{Mixtures with small MNPs}

\subsubsection{Structure and phase diagram}

We start by considering LC-MNP systems with "small” MNPs, that is, $\sigma_{\mathrm{s}}^{\star}=\sigma_{\mathrm{s}} / \sigma=1 / 4$ [31]. This size ratio is realistic, e.g., for colloidal systems of pigment nanorods (typical width $40 \mathrm{~nm}$ ) and magnetic spheres (diameter approx. $10 \mathrm{~nm}$ ) [12]. In Ref. [31], we have examined the self-assembly and equilibrium dynamics of the MNPs within the LC matrix which can be either isotropic or orientationally ordered.

The topology of the phase diagram that has been obtained is similar to the corresponding of the pure GB system. It contains three fully miscible phases, namely an isotropic (I), a nematic $(\mathrm{N})$ and a smectic- $\mathrm{B}(\mathrm{SmB})$ phases. This already indicates that the MNPs do not exert a strong perturbation on the LC matrix at $\sigma_{\mathrm{s}}^{\star}=1 / 4$. The volume fraction ratio of the GB rods over MNP species is $\phi_{\mathrm{r}} / \phi_{\mathrm{s}} \cong 780$. This implies that we essentially examine the influence of the LC matrix on the MNPs rather than the opposite.

Under the conditions considered, the MNPs form clusters within the LC matrix, see Figure 1. The types of the clusters can be classified into four categories: i) chain-like clusters that consist of at least three particles (here, the end-chain particles have only one bond), ii) ring-like clusters (that form loops) in which all the particles have at least two bonds, iii) branched clusters (for which at least one particle of the cluster has three or more bonds with the other particles), and iv) "free", non-bonded particles or pairs of MNPs. We have found that the orientational state of the LC matrix (either isotropic or 

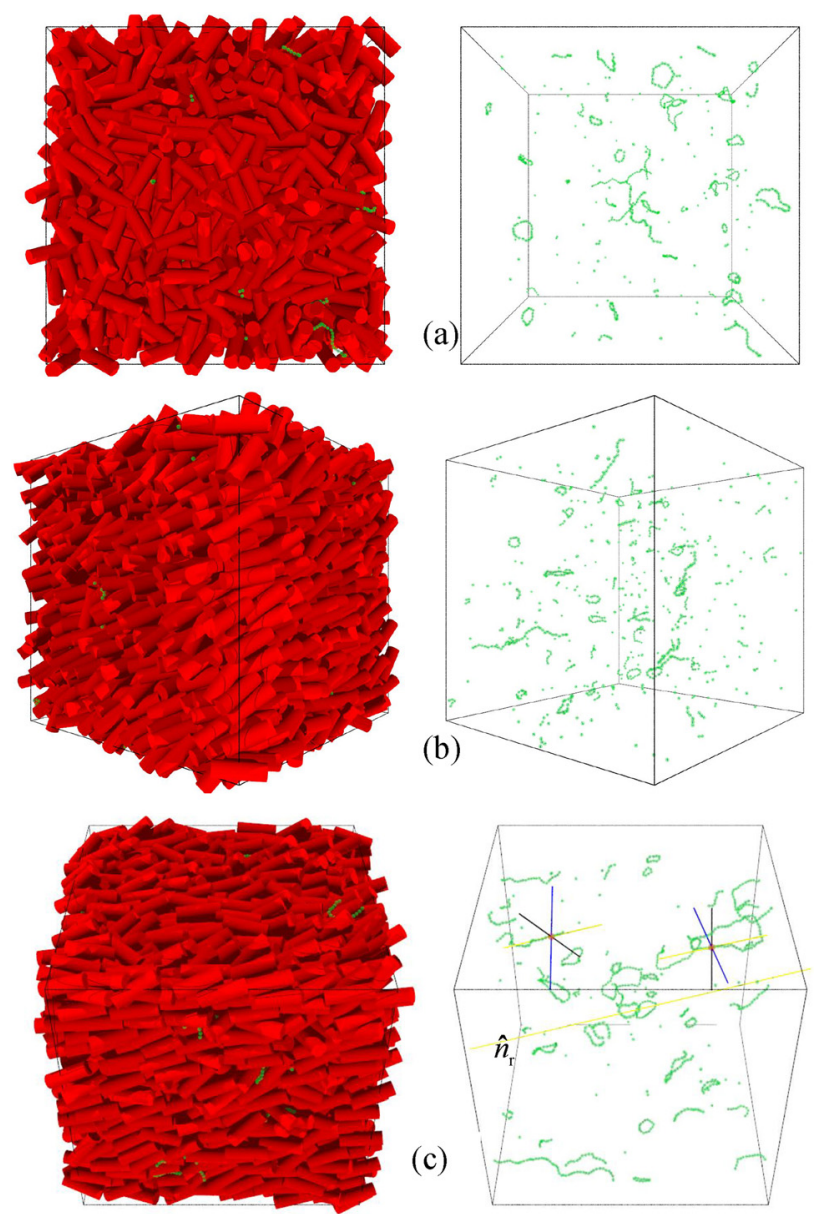

Figure 1: Representative snapshots of a GB-MNP mixture with $\sigma_{\mathrm{s}}^{*}=0.25$ and (concentration of rods) $x_{\mathrm{r}}=0.8$ in various states [(reduced temperature, number density)]: (a) isotropic state at $\left[\left(T^{*}, \rho^{*}\right)=(1.2,0.34)\right]$ with $\lambda=2.5$, (b) uniaxial nematic $\left(N_{u}\right)$ state at $\left[\left(T^{*}, \rho^{*}\right)=(1.4,0.44)\right]$ with $\lambda=2.14$ and (c) uniaxial nematic $\left(\mathrm{N}_{\mathrm{u}}\right)$ at $\left[\left(T^{*}, \rho^{*}\right)=(1.2,0.44)\right]$ with $\lambda=2.5$. The direction of $\hat{\mathrm{n}}_{\mathrm{r}}$ and the principal axis frames of a ring and a chain are also shown. For clarity, the rod species have been removed from the simulation box (right column). Reprinted from Ref. [31].

liquid crystalline) does not affect the type or the size of these clusters. Their size is mainly determined by the dipolar coupling parameter $\lambda$ that for these systems takes values greater than 6 . Similar types of clusters have been observed in monodispersed MNPs systems [44].

Still, an important finding for the mixed system is that the matrix in its nematic state promotes some degree of orientational order of the clusters (e.g., of the chains or rings). We have quantified these features by appropriate local order parameters [31]. 
Further, the observed alignment of the clusters along the director of the LC phase is enhanced at lower temperatures (i.e., larger values of $\lambda$ ), where more "tight" clusters are formed. Another interesting finding is that even though the matrix is uniaxial, the clusters (due to their non-uniaxial shape) do not rotate freely around the director of the phase.

\subsubsection{Impact of an external field, relation to experiment}

Starting from the LC-MNP model system with $\sigma_{\mathrm{s}}^{\star}=1 / 4$, we have also investigated the impact of a uniform, external magnetic field on the structural behavior of the mixture. This study was motivated by a collaboration with experiments [12]. The latter had revealed that the magnetic field can induce orientational order to the matrix of nonmagnetic rodlike particles (in this case, pigment rod) even when this matrix is isotropic in the field-free case. To understand this behavior on a particle level, we have employed MD simulations.

The MD simulation results not only describe qualitatively the behavior of the experimental system, but also provide a "bottom up" connection between the macroscopic properties (e.g., global orientational order parameters and birefringence) and microscopic inter-particle correlations. In particular, we have found that the MNPs self-assemble into 'rodlike' entities (i.e., chain-like clusters) along the direction of the magnetic field. These chain-like clusters hinder the rotational motion of the nonmagnetic rods into perpendicular conformations with respect to the direction of the magnetic field. As a result, indirectly, nematic ordering is induced to the non-magnetic rods even though their zero-field state is an isotropic one. The main ingredient for the alignment of the rodlike species are, on the one hand, the size of the clusters (i.e., the length of the rodlike entities, which depends on $\lambda$ ), and on the other hand, the number per volume of the chain-like clusters [12]. In conclusion, our MD simulations clearly demonstrated the (hitherto only suspected) microscopic mechanisms that drive the macroscopic orientational behavior of such real systems. We note, however, that our model is not appropriate to describe suspensions of large, micron-sized magnetic particles in low molar mass liquid crystals (see for example Ref. [13]), where the MNPs induce strong distortions of the LC director field. For such systems theoretical approaches have been developed in which the LC is usually considered as continuum medium [45].

\subsection{Mixtures with similar size components}

Using MC simulations, we have also explored the structure and self-assembly of mixtures in which the diameter of the magnetic spheres is equal or greater than the width of the GB rods $[29,30]$. The main motivation here was to understand the role of the rod-sphere size ratio on the stability of formed structures (e.g., rings and branched 
clusters), as well as the collective ordering of the MNPs and their influence on the LC matrix.

In the present section we focus on the case $\sigma_{\mathrm{s}}^{\star}=1.0$ (MNP diameter equal to the width of the GB rods). The coupling parameter $\lambda$ takes values between 3.5 and 15 , corresponding to strongly coupled MNPs. As a reference system we also studied a mixture of GB rods with non-magnetic spheres. This reference system displays fully miscible, isotropic, nematic and smectic-B phases without any demixing or phase separation. Our MC simulations for the full system (LC matrix mixed with magnetic spheres) reveal a state diagram similar to the reference system. The volume fraction ratio of the GB rods over MNPs is $\phi_{\mathrm{r}} / \phi_{\mathrm{s}} \cong 12$. Interestingly, the tentative phase boundaries of the orientational ordered states (e.g., the nematic) are generally shifted towards higher temperatures (as compared to the reference system) indicating the enhancement of orientational order, despite the absence of an external magnetic field. An illustration of the microscopic structure of MNPs in various LC states is given in Figure 2. In the isotropic state, the MNPs self-assemble into (branched) worm-like chains that extend all over the simulation cell without orientational order. Note that due to the large value of $\lambda$, we do not observe the formation of finite-sized clusters. As the LC matrix undergoes a transition from the isotropic to the nematic state, the chains spontaneously "unwrap" transforming to essentially straight chains oriented along the nematic director. The dipole moments of the MNPs adopt a "head-to-tail" configuration forming ferromagnetic chains. Nevertheless, the net magnetization is zero since the chains adopt random "up-down” configurations.

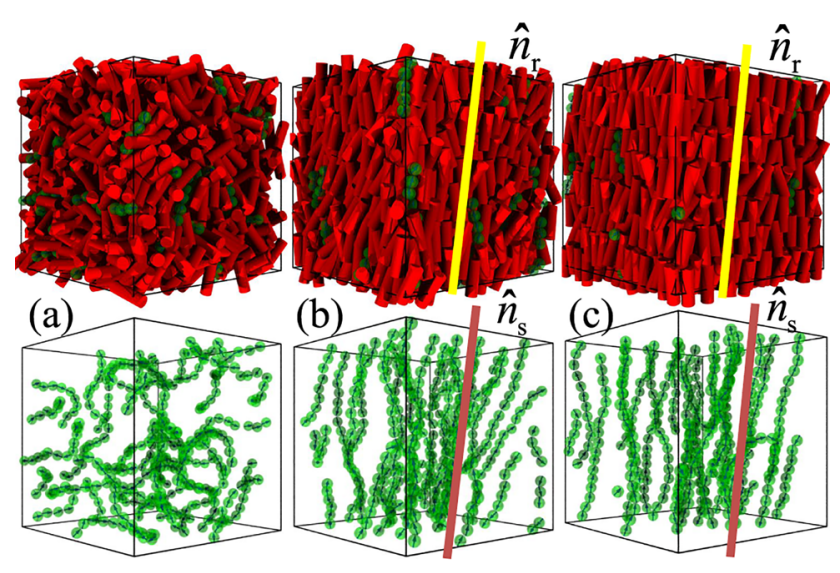

Figure 2: Representative simulation snapshots for mixtures with $\sigma_{\mathrm{s}}^{*}=1$ and (concentration) $x_{\mathrm{r}}=0.8$ in various states [(reduced temperature, number density)]: a) the isotropic $(I)$ state $\left[\left(T^{*}, \rho^{*}\right)=(1.1,0.34)\right]$ with $\lambda=2.73$, b) uniaxial nematic $\left(\mathrm{N}_{\mathrm{u}}\right)$ state $\left[\left(T^{*}, \rho^{*}\right)=(1.2,0.40)\right]$ with $\left.\lambda=2.5, \mathrm{c}\right)$ uniaxial smectic-B $(\mathrm{SmB})$ state $\left[\left(T^{*}, \rho^{*}\right)=(0.9,0.40)\right]$ with $\lambda=3.33$. In the bottom parts only the MNPs are shown for clarity. The directors $\widehat{\boldsymbol{n}}_{\mathrm{r}}$ and $\widehat{\boldsymbol{n}}_{\mathrm{s}}$ are indicated by thick lines. Reprinted from Ref. [29]. 
Taken altogether, the ferromagnetic chains stabilize the LC ordering (as demonstrated, for example, by the shift of the isotropic-to-nematic (I-N) phase transition towards higher temperatures) [29]. Vice versa, the LC matrix stabilizes the alignment of the chains along a common direction (i.e., the director of the nematic state), which is absent in pure MNPs fluids at the densities considered here. Thus, the LC matrix has a similar effect as an external field regarding the orientational ordering of the chains. The main difference is that an external magnetic field not only aligns the chains, but can also cause them to point along the same direction, yielding a globally ferromagnetic state.

The results discussed so far already indicate that the self-assembly of the MNPs, as well as their interplay with the LC matrix, are sensitive to the diameter ratio of the spheres relative to the GB rods' width. In what follows we focus on this effect for size ratios $\sigma_{\mathrm{s}}^{\star}>1.0$.

\subsection{Mixtures with larger MNPs}

For a mixture of GB rods and MNPs with diameter $\sigma_{\mathrm{s}}^{\star}=1.5$ and volume fraction ratio of GB rods over MNPs $\phi_{\mathrm{r}} / \phi_{\mathrm{s}} \cong 3.54$, the LC matrix exhibits isotropic, nematic and highly interdigitated smectic states. The nematic state is significantly promoted as compared to the system with $\sigma_{\mathrm{s}}^{\star}=1$.0. Nevertheless, the degree of alignment of the ferromagnetic chains is smaller. Furthermore, at lower temperatures, a layered state with interdigitated configuration of the rods is formed. This is in contrast to the system with $\sigma_{\mathrm{s}}^{\star}=1.0$, in which well-defined periodic layers are formed. This indicates that the MNPs exert an appreciable perturbation to the (partially) positionally ordered liquid crystalline state of the matrix.

By further increasing the diameter of MNPs to $\sigma_{\mathrm{s}}^{\star}$ greater than 1.7, significant changes occurs in the morphology of the system, see Refs. [29, 30]. In particular, we refer to systems with $\sigma_{\mathrm{s}}^{\star}=1.7$ and $\sigma_{\mathrm{s}}^{\star}=2.0$ with volume fractions $\phi_{\mathrm{r}} / \phi_{\mathrm{s}} \cong 2.44$ and $\phi_{\mathrm{r}} / \phi_{\mathrm{s}} \cong 3.4$, respectively.

In these systems the perturbation of the MNPs to the LC matrix is so strong, that the LC matrix does not induce any significant alignment of the ferromagnetic chains in the nematic state. In addition, the MNPs destabilize the conventional smectic order (e.g., the smectic B state). For these systems, at lower temperatures, a lamellar structure occurs with alternating rod-rich and MNP-rich layers (microphase separation). The MNPs self-assemble into snake-like chains in an antiparallel manner, therefore, no net magnetization occurs. The most striking finding is that the mean alignment of the chains (i.e., the MNPs director) is perpendicular to the director of the rods giving rise to a biaxial lamellar state $\left(\mathrm{L}_{\mathrm{b}}\right)$ (see Figure 3 ). This biaxial LC-MNP system differs from conventional biaxial LCs, that are generated by the shape of the particles [46] or from biaxial mixtures, of rods and disks [47]. In the case of LC-MNP systems, the biaxiality stems from the spontaneous organization of the ferromagnetic chains (of non-LC 


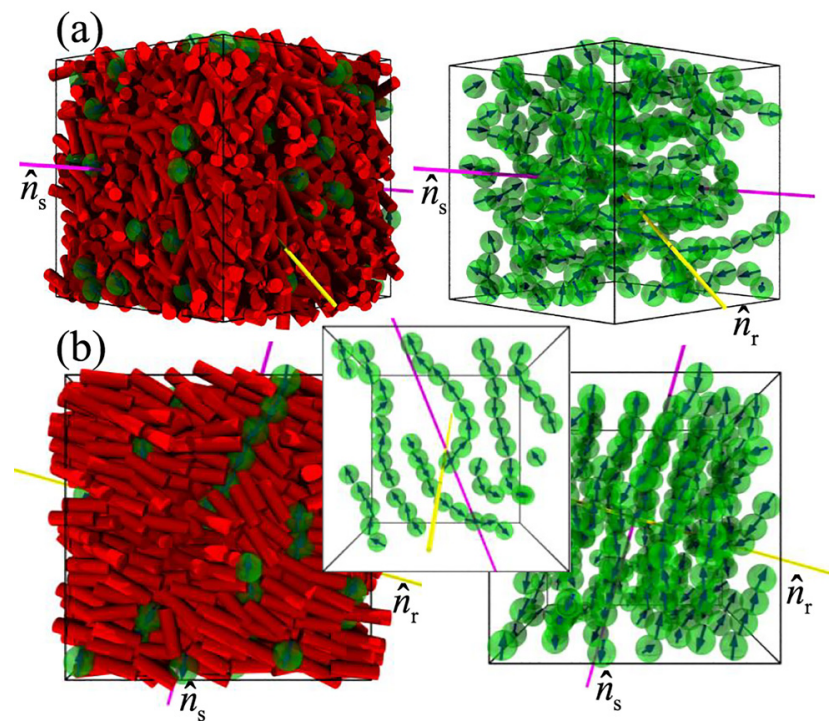

Figure 3: Representative simulation snapshots for mixtures with $\sigma_{\mathrm{s}}^{*}=2$ and (concentration) $x_{\mathrm{r}}=0.9$ in various states [(reduced temperature, number density)] a) the biaxial nematic $\left(\mathrm{N}_{\mathrm{b}}\right)$ state $\left[\left(T^{*}, \rho^{*}\right)=(1.8,0.338)\right]$ with $\lambda=1.67$ and $\left.b\right)$ the biaxial lamellar state $\left[\left(T^{*}, \rho^{*}\right)=(1.2,0.338)\right]$ with $\lambda=2.5$. On the right sides only the MNPs are shown for clarity. Part b) additionally includes a snapshot of the structure in one dipolar layer. The directors $\widehat{n}_{r}$ and $\widehat{n}_{s}$ are indicated by thick lines. Reprinted from Ref. [29].

particles) within the LC matrix. The biaxial ordered hybrid also exhibits strong anisotropic sensitivity to external homogeneous magnetic field [30]. Upon applying a magnetic field to the LC-MNP matrix with $\sigma_{\mathrm{s}}^{\star}=2.0$ in an otherwise isotropic state, orientational ordering is induced to the rodlike species. Interestingly, the director of the nematic state is perpendicular to the external field. On the other hand, the ferromagnetic chains align parallel to the direction of the magnetic field. Hence, a biaxial state can be induced in an initially isotropic state upon applying a homogeneous magnetic field. This is in contrast to systems with $\sigma_{\mathrm{s}}^{\star}$ less than 1.5, where the field induces a nematic state with the LC director being parallel to that of the ferromagnetic chains.

From a physical point of view, one would expect that the complex morphologies also affect material properties such as light propagation and thermal transport in corresponding real soft matter magnetic systems. Recently, we have examined [38] the light propagation through MNPs in LC matrices. We have found that LC-MNP hybrids exhibit a strong magnetochiral dischroism (MCD) in a wide range of light wavelengths (in the visible regime from 500 to $750 \mathrm{~nm}$ ). This behavior is generated by the significant changes in the magnetic MNP self-organization as the LC matrix undergoes a transition from an isotropic to a nematic state upon applying an external homogeneous magnetic field. 


\section{Equilibrium dynamics of mixtures of liquid crystals and magnetic nanoparticles}

So far, we have focused on purely structural effects. We now turn to the equilibrium dynamics in the absence of a magnetic field or shear flow, focusing on the translational diffusion. It is well established by computer simulation studies (see, e.g., [43, 48, 49]) that pure magnetic dipolar fluids, even at low densities, can show interesting diffusional dynamics which strongly correlates with the structures they form. Therefore, and in view of the novel structures formed in our composite systems, we have performed various MD simulation studies [31, 32] to examine the equilibrium dynamics of LC-MNP systems with size ratios $\sigma_{\mathrm{s}}^{\star}=1 / 4$ (Section 3.1) and $\sigma_{\mathrm{s}}^{\star}=1.0$ (Section 3.2). Our results show that both, the orientational state of the matrix and the dipolar coupling strength, $\lambda$ play important role on the translational dynamics.

\subsection{Mixtures with similar size components}

We start by discussing the case $\sigma_{\mathrm{s}}^{\star}=1.0$, which we have investigated in most detail [32]. To elucidate the translational dynamics, we have analyzed the mean square displacement (MSD) of different species at different temperatures and densities. With changing temperatures, the strength of the dipolar coupling, $\lambda$, also changes which affects the size of dipolar chains [43]. Therefore, it is expected that the dynamics of the dipolar particles will becomes slower as the temperature is decreased. In fact, for pure dipolar fluids, a subdiffusive behavior is observed at intermediate times which then crosses over to the diffusive behavior at late times [48]. The situation in present case is, however, different: here, apart from the different chain sizes at different temperature, the LC matrix provides an anisotropic dense medium, which significantly modifies the dynamics of the MNPs [32].

We first consider the MSDs of the MNPs in the isotropic phase, i.e., at the temperatures and densities where the LC host matrix remains in the isotropic phase. The MSDs of the MNPs in the isotropic phase are plotted in Figure 4 for $\rho^{\star}=0.3$ and various values of $\lambda$. For all $\lambda<11.25$, the MSDs show three different regimes, ballistic at short times, subdiffusive at intermediate times and diffusive at long times. At low $\lambda$, the subdiffusive regime is rather short, however, as $\lambda$ is increased the subdiffusive regime grows, and for $\lambda>9.0$ at $\rho^{\star}=0.3$, it spans the entire simulation time window. The subdiffusive behavior in this case is attributed to the combined effect of the complex environment by the LC matrix, on one hand, and the chain forming tendency of the dipolar fluids, on the other hand. The exponent of the MSD in the subdiffusive regime is obtained as $\sim 0.6$ [32].

In the nematic phase of the LC host matrix, the MSD of the MNPs can be decomposed into components parallel and perpendicular to the nematic director, pf the MNP 


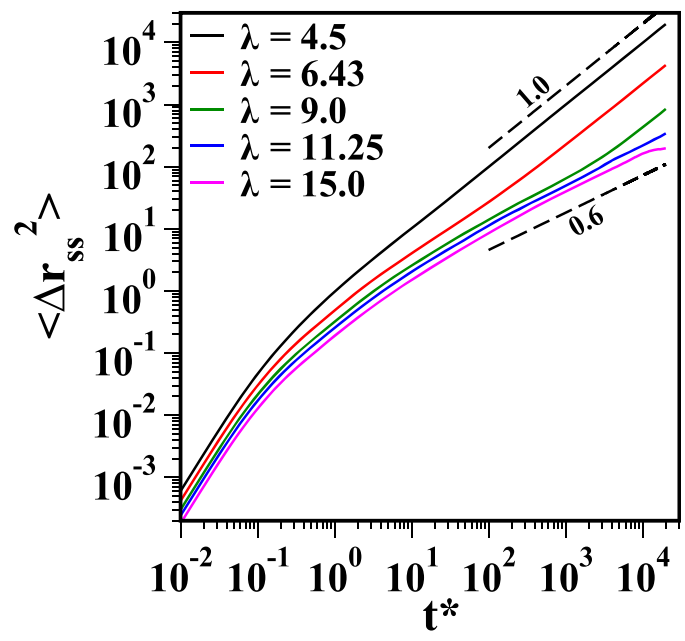

Figure 4: MSD of MNP in the isotropic regime for $\rho^{*}=0.3$ at $\lambda=4.5,6.43,11.25$, 15.0. At low $\lambda$, the MSD has a slope of 1.0 at large $t$ (shown by black dashed line). At large $\lambda$, subdiffusive behavior is observed with an exponent 0.6. Reprinted from Ref. [32].

chains, $\widehat{\boldsymbol{n}}_{s}$. We fix $\lambda=11.25$ (i.e., $T^{\star}=0.8$ ) and investigate the MSD for different $\rho^{\star}$. The reason behind such a choice of $\lambda$ is that at this value, the MSDs of the MNPs remain subdiffusive during the entire simulation time window. Figure 5(a) shows the component of the MSD of the MNPs parallel to $\widehat{\boldsymbol{n}}_{s}$. One observes an initial subdiffusive increase with time (reminiscent of the isotropic phase), which then crosses over to a normal diffusive behavior at long times (for all considered densities). The component of the MSD perpendicular to $\widehat{\boldsymbol{n}}_{s}$, plotted in Figure 5(b), remains subdiffusive even at long times. The exponent of subdiffusion is $\sim 0.5$, which is smaller than the observed exponent in the isotropic regime for the total MSD (at the same $\rho^{\star}$ and $\lambda$ ). The inset in Figure 5(b) illustrates the trapping of MNP chains in the transient cylindrical cavities formed by the LC matrix in the nematic phase. This leads to a slowing down of the
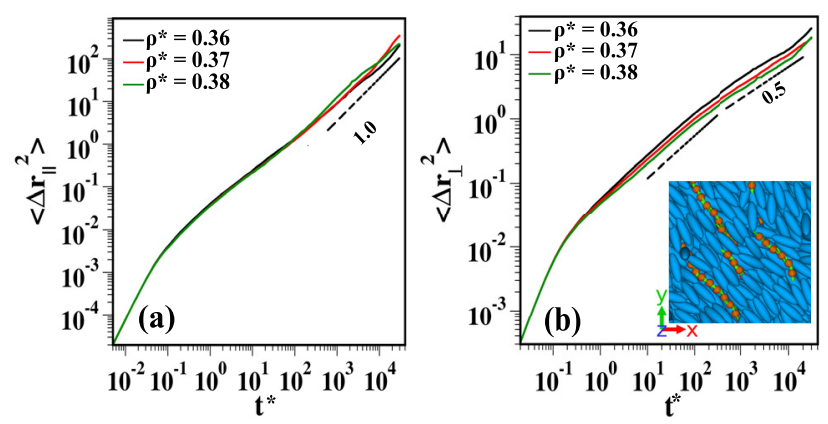

Figure 5: (a) The component of MSD parallel to the $\widehat{\boldsymbol{n}}_{s}$ for MNPs at the same values of $\rho^{\star}$ and $\lambda$ as in (a). The black dashed line shows a slope 1.0. (b) The component of the MSD of the MNPs perpendicular to the $\widehat{\boldsymbol{n}}_{s}$ at $\rho^{*}=0.36,0.37,0.38$ and $\lambda=11.25$. The inset shows a top view of the snapshot for $\rho^{*}=0.37$ and $\lambda=11.25$. The MSD shows a subdiffusive behavior with exponent 0.5. Reprinted from Ref. [32]. 
translational dynamics of the MNPs in the direction perpendicular to the nematic director [32].

The trapping scenario is further clarified by investigating the velocity autocorrelation functions (VACF) for the MNPs. In the nematic phase, the component of the VACF for the MNPs parallel to the nematic directior, plotted in Figure 6(c) (shown by red dashed line) decays faster than the component in the perpendicular direction (represented by green dot-dashed line). The oscillations after the smooth initial decay are present in both the components. The oscillations in the parallel component arise due to the strong dipolar interactions which force the particles to remain in the chain. In the present case, the oscillations in the perpendicular component are the result of the confinement of the MNP chains in a narrow cylindrical channel formed by the LC matrix.

We also note that the dynamics of the LC particles remains unaffected by the MNPs. The MSD of the LC particles shows a diffusive behavior at long times in the isotropic phase $[32,50]$. In the nematic phase, the components of the MSD of the LC particles remains diffusive in both, the direction parallel and perpendicular to the nematic director. Additionally, in the nematic direction the diffusion coefficient shows a nonmonotonic behavior as a function of the density. Such a non-monotonic behavior occurs due to the faster movement of the LC particles along the director in the low density range of the nematic phase $[50,51]$.

\subsection{Mixtures with small MNPs}

Finally, we briefly consider the case $\sigma_{\mathrm{s}}^{\star}=1 / 4$. Here, we find that the LC matrix in its nematic state not only induces orientational ordering of the clusters (see also

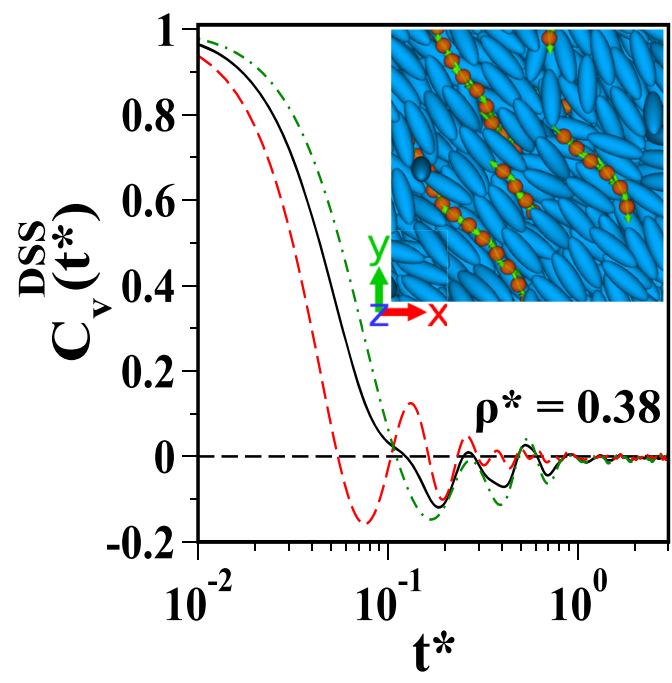

Figure 6: The VACF for MNP in the nematic regime at $\rho^{*}=0.38$ and $\lambda=11.25$. The red dashed and green dotdashed lines represent the function $C_{v_{\|}}(t)$ and $C_{v_{\perp}}(t)$. The inset shows a top view of the snapshot for $\rho^{*}=0.37$ and $\lambda=11.25$. Reprinted from Ref. [32]. 
Section 2.2.1), but also promotes their motion along the director [31]. This is indicated by the fact that the long-time diffusion coefficient is largest in this direction. This is different at short times, where the MNPs travel faster perpendicularly to the nematic director. Regarding the impact of coupling, we find that the self-diffusion coefficient of the MNPs decreases by increasing $\lambda$, as the percentage of the "free" magnetic particles decreases. This gives place to the formation of larger clusters that slow down the mobility of the MNPs. Further, the so-called "non-gaussian" parameter that is zero in the perfect ballistic or diffusive regime, shows deviation from zero indicating nontrivial behavior of the MNPs. In summary, our MD simulations indicate the presence of complex translational dynamics of the MNPs that depend on the orientational state of the matrix, the corresponding MNP cluster formation, and on the size ratio.

\section{Rheology of LC-MNP mixtures}

While the equilibrium structure and dynamics of LC-MNP mixtures is important and quite intriguing, many applications of such soft hybrid systems, as well as some experiments, actually involve nonequilibrium conditions, particularly shear flow. Taking this as a motivation, we here present results from nonequilibrium molecular dynamics (NEMD) simulations for model mixtures composed of GB rods and spherical MNPs with $\sigma_{\mathrm{s}}{ }^{\star}=1.0$ (Section 4.1), as well as elongated MNPs (Section 4.2), in planar Couette shear flow.

\subsection{Spherical MNPs}

\subsubsection{The flow curve}

The rheological behavior of the LC-MNP mixtures is investigated by applying a steady shear (via Lee-Edwards boundary conditions) with a constant shear rate $\dot{\gamma}$. We calculate the evolution of the stress, $\sigma_{x z}$, as a function of strain. After reaching a steady state, the corresponding stress $\left(\sigma_{x z}^{s s}\right)$ can be plotted as a function of the shear rate, yielding the socalled flow curve. Figure 7(a) shows the flow curves for the LC-MNP mixture for different $\lambda$. For all values of $\lambda$ except $\lambda=11.25$, we observe a crossover from "Newtonian" behavior, where $\sigma_{x z}^{s s}$ is linearly proportional to $\dot{\gamma}^{\star}$, to "non-Newtonian" behavior, where $\sigma_{x z}^{s s}$ varies in a power-law manner as a function of $\dot{\gamma}^{*}$. This transition occurs at a "critical" shear rate, which is approximately given by $\dot{\gamma}_{c}^{\star}=0.02$ [33].

At shear rates $\dot{\gamma}^{*} \gtrsim \dot{\gamma}_{c}^{*}$ the stress varies as $\sigma_{x z}^{s s} \propto \dot{\gamma}^{* n}$ for all values of $\lambda$, where the power-law exponent is $n \approx 0.8$. This exponent, also called flow index, characterizes the non-Newtonian behavior of the LC-MNP mixture. Specifically, the fact that for $\dot{\gamma}^{\star} \geq \dot{y}_{c}^{*}, n$ is smaller than one, indicates a shear thinning. This behavior is also reflected by the average viscosity, defined as $\eta=\sigma_{x z}^{s s} / \dot{y}^{\star}$, which is plotted in Figure 7(b) as a function of 

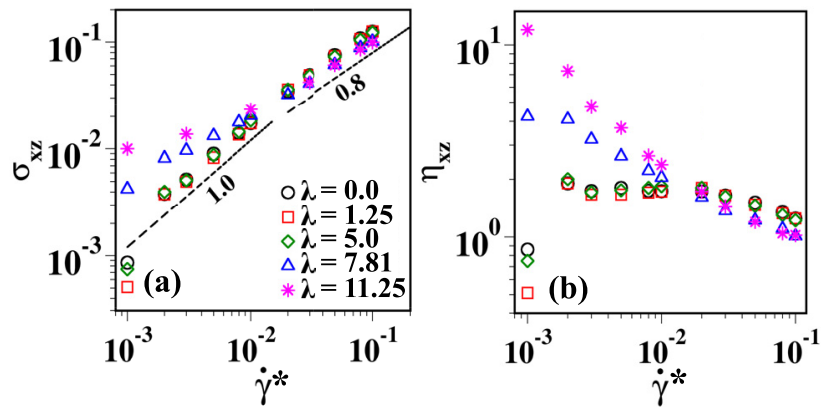

Figure 7: (a) The flow curve for the LC-MNP mixture at $\rho^{*}=0.34, T^{*}=0.8$ and $\lambda=0.0,1.25,5.0,7.81$, 11.25. Dashed lines represent slopes 1.0 and 0.8. (b) Average viscosities $\eta_{x z}$, obtained from $\sigma_{x z}^{s s}$, as a function of $\dot{\gamma}^{*}$ for the same $\lambda$ values as in (a). Here, different symbols represent the same $\lambda$ value as in (a). Reprinted from Ref. [33].

$\dot{y}^{\star}$. For a wide range of complex fluids such as microgels, foams, and emulsions, the exponent $n$ is considered to be a "material parameter" that weakly depends on the temperature and density [52].

\subsubsection{Microstructure under shear}

The nonlinear features observed in the flow curves discussed in the previous subsection already suggest profound structural changes. In the present section we focus, in particular, on the chain formation of magnetic particles. To this end we consider mixtures at $\lambda=5.0$ and $\lambda=11.25$, where the non-Newtonian behavior is particularly pronounced. Before we move ahead with the analysis, it should be noted that here in our LC-MNP mixture, the number density of the MNP is rather small $\left(\rho_{M N P}^{\star}=0.068\right)$. In pure MNP fluids, at such low densities, the MNPs form chains with head-to-tail ordering of neighboring particles and the size of the chains depending on $\lambda$. These MNP chains are isotropically distributed, that is, there is no long-range orientational order [40, 43]. In our previous study of a LC-MNP mixture, we have shown that in the isotropic phase (such as one considered here at $\rho^{\star}=0.34$ ), the MNPs form isotropically distributed chains of significant length if $\lambda \geq 6.0$ [32]. Therefore, we expect that in equilibrium, sizes of the MNP chains should be rather small for $\lambda=5.0$ and relatively larger for $\lambda=11.25$.

We find that at the low shear rate, $\dot{\gamma}^{\star}=5 \times 10^{-3}$, LCs do not show the shear-induced alignment, see Figure 8(a). On the contrary, at the high shear rate, $\dot{\gamma}^{*}=10^{-1}$, the LCs display a shear-induced alignment in the steady-state, see Figure $8(\mathrm{~b})$. The MNP, plotted in Figure 8(c), (d), show no alignment in the direction of the shear at any of the shear rates considered [33].

In contrast to $\lambda=5.0$, at large $(\lambda=11.25)$ the equilibrium configuration is characterized by large MNP chains. In the presence of shear, these MNP chains align with the 

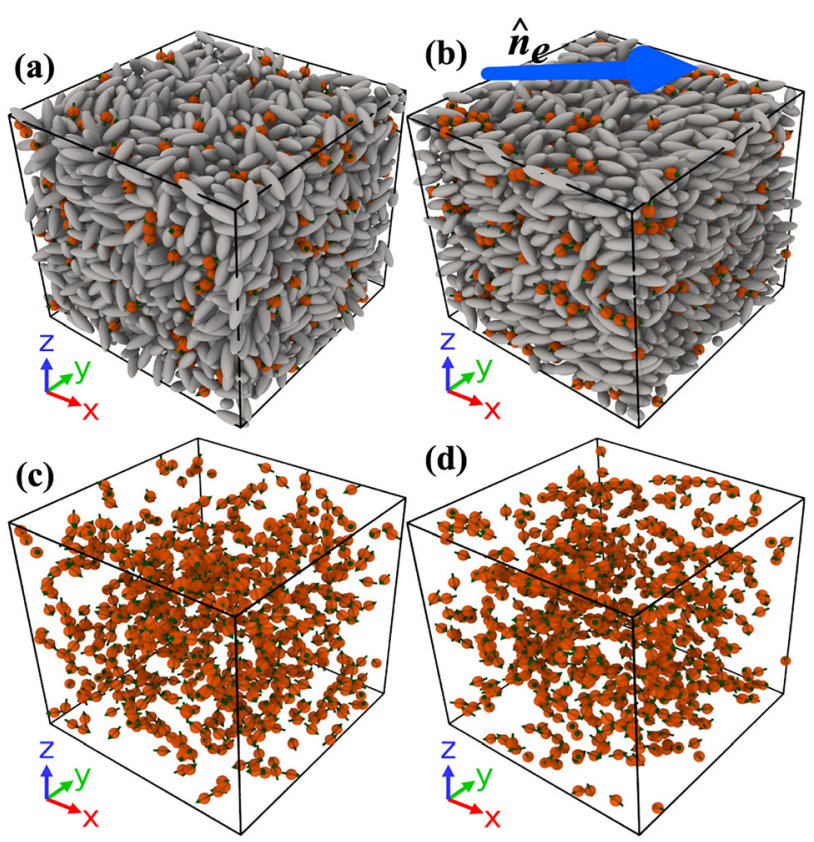

Figure 8: Snapshots of the LC-MNP mixture for $\lambda=5.0, \rho^{*}=0.34$ and $T^{*}=0.8$ under shear. (a) and (b) illustrate the structure in the steady state $\left(\dot{\gamma}^{*} t^{\star}=15.0\right)$ for $\dot{\gamma}^{*}=5 \times 10^{-3}$ and $10^{-1}$, respectively. The snapshots (c) and (d) show the MNPs alone at the parameters corresponding to (a), and

(b) respectively. Reprinted from Ref. [33].

shear direction at both shear rates considered (see Figure 9(c), (d)). The response of the LCs depends on the shear rate: while the LC system at $\dot{\gamma}^{\star}=5 \times 10^{-3}$ (Figure 9(a)) displays weak alignment, the ordering at $\dot{\gamma}^{\star}=10^{-1}$ is more significant, see Figure 9(a). Here we already note that the observed shear-induced ordering of the two species is consistent with previous simulation studies on pure LCs [53-55] and pure dipolar fluids [56]. The difference in the present case is that the two components strongly influence each other.

These shear-induced changes in the microstructure are better quantified by investigating the nematic order parameters at different shear rates in the steady state for both the species of the mixture. The nematic order parameters are defined as the largest eigen value of the ordering matrices for the LCs and the MNPs. In Figure 10(a), we plot the steady-state nematic order parameters $S^{\text {ss }}$ for the LC $\left(S_{e}\right.$ black circles $)$ and the MNP $\left(S_{s}\right.$ red squares) for $\lambda=5.0$ at different shear rates. We recall that at $\lambda=5.0$, the flow curve reveals both, a Newtonian regime $\left(\dot{\gamma}^{\star}<\dot{\gamma}_{c}^{\star}\right)$ and a non-Newtonian regime at high shear rates. As seen from Figure 10(a), the MNP do not develop any pronounced nematic ordering throughout the range of the shear rates considered. This is different for the LCs. In the Newtonian regime, the LCs do not show shear-induced ordering while at high shear rates, significant (para-)nematic ordering is observed. To this end 

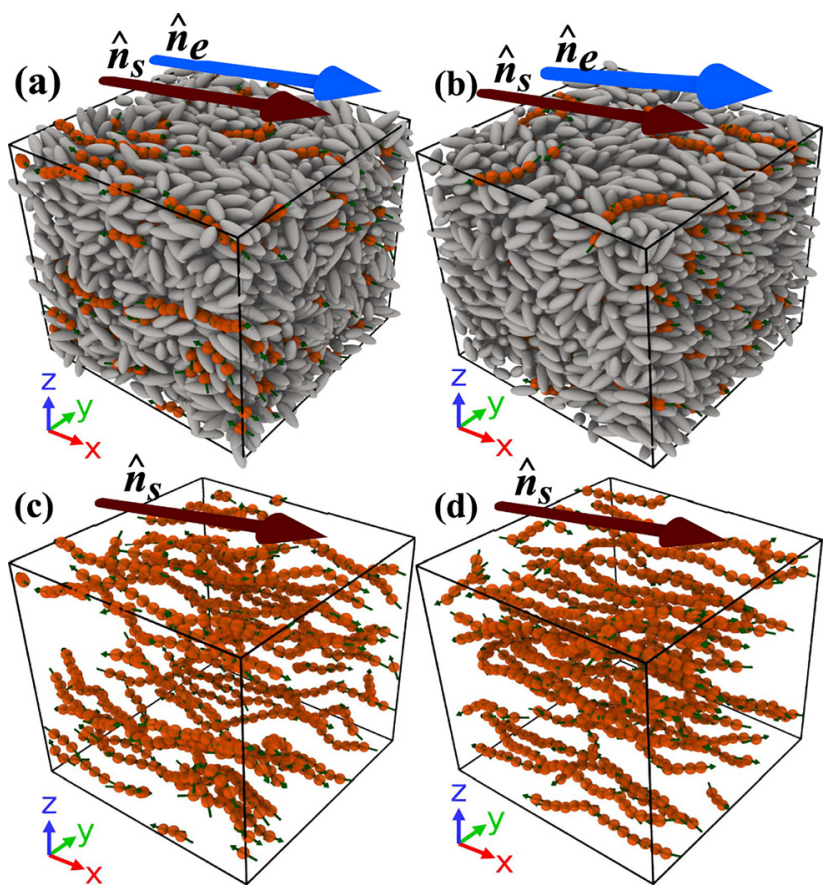

Figure 9: Snapshots of the LC-MNP mixture for $\lambda=11.25, \rho^{*}=0.34$ and $T^{*}=0.8$ under shear. (a) and (b) illustrate the structure in the steady state $\left(\dot{\gamma}^{*} t^{\star}=15.0\right)$ for $\dot{\gamma}^{*}=5 \times 10^{-3}$ and $10^{-1}$, respectively. The snapshots (c) and (d) show the MNPs alone at the parameters corresponding to (a), and

(b) respectively. Reprinted from Ref. [33].

we note that the value of $S_{e}$ related to the equilibrium I-N transition is 0.43 according to the Marier-Saupe theory [45]. This value of $\left(S_{e}=0.43\right)$ is represented by the horizontal light-blue dashed line in Figure 10. One sees that this (equilibrium) value is approached and finally exceeded when the shear rate becomes larger than $\dot{\gamma}_{c}^{\star}$. We conclude that the non-Newtonian regime of the flow curve at $\lambda=5.0$ is accompanied by orientational ordering of the LCs, but not the MNPs.

The situation at large $\lambda$, shown in Figure 10(b), is different. Here, the LC matrix exhibits a significant degree of ordering already at the lowest shear rate, $\dot{\gamma}^{\star}=10^{-3}$. Moreover, the nematic order parameter of the MNP is even larger. We understand these properties, which are in marked contrast to those observed at $\lambda=5.0$, as a consequence of the pronounced chain formation of the MNP at $\lambda=11.25$, see Figure 9(c), (d). Due to the strong correlation within the chains, these form rather stiff and long objects which align in the shear flow. The alignment of the chains, in turn, enhances the alignment of the non-magnetic LC particles, leading to relatively large values of $S_{e}^{s s}$.

Upon increase of the shear rate, the nematic order parameter of the MNP remains essentially constant in the range of the shear rates considered. For pure dipolar fluids 

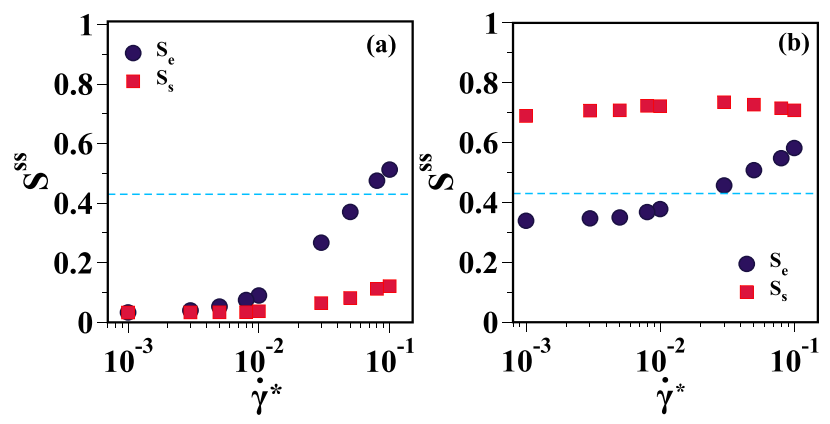

Figure 10: (a) Variation of the nematic order parameter in the steady state for $\lambda=5.0$ for the LC $\left(S_{e}\right)$ and the MNPs $\left(S_{s}\right)$ as a function of $\dot{\gamma}^{*}$. The horizontal blue dashed line represents the critical value of the nematic order parameter at which I-N transition is observed. (b) Variation of the nematic order parameters for the LC $\left(S_{e}\right)$ and the MNPs $\left(S_{s}\right)$ in the steady state for $\lambda=11.25$ as a function of $\dot{\gamma}^{*}$. Reprinted from Ref. [33].

under shear, it has been observed that the nematic order parameter decreases at high shear rates due to breaking of the chains [56]. In the present study, we do not observe such a behavior as the maximum shear rate is restricted to $\dot{y}^{*}=0.1$.

In summary, these results for mixtures with spherical MNPs indicate that the flow behavior is strongly affected by the strength of dipolar coupling among the MNPs. This property may find appealing technical applications in the situations where tunable flow properties are desired.

\subsection{Elongated MNPs}

In many real examples of LC-MNP mixtures, the MNPs are spherical (and hence different from the LCs). However, they can also be shape-anisotropic (which often goes along with a strong size difference relative to the $\mathrm{LC}$ particles) $[5,8,15,16]$. The impact of non-spherical shape is indeed a timely issue in view of recent experimental realizations $[9,13,17,18]$, which have also motivated a number of analytical and numerical studies [57-60].

In the present section we discuss mixtures where the shape and size of the MNPs are identical to those of the LC particles. Clearly, this is a somewhat idealized situation compared to real systems. On the other hand, this choice simplifies a computational study because it eliminates effects induced by shape and size mismatch (see Refs. [61-66] for some examples).

The aforementioned recent studies involving nonspheriacl MNPs [57-60] are concerned with the effect of a magnetic field on the structural and optical properties. Here we rather focus on the response of the aforementioned mixtures to the mechanical perturbation (shear) in the presence of an external magnetic field. One should note that 
there is a key difference between optical and mechanical responses; unlike in the optical case, where light is transmitted through the sample without altering the structure, shearing itself can lead to structural changes [54, 67-70]. Therefore, the structure and rheology of such mixtures are affected by the interplay between the ordering induced by shear (see Ref. [71] and references therein) and the ordering induced by the magnetic field.

\subsubsection{Model and simulation set-up}

To isolate the role of the magnetic field-induced orientation of the MNPs on the structure and rheology, we consider a mixture where not only the shape and size of the MNPs and LCs are identical (both are described by GB particles with aspect ratio 3:1), but also they interact via the same pair potential. In this case, only the directions of the MNPs are coupled to the external magnetic field, and this coupling distinguishes the MNPs from the LCs. It is worth mentioning that the similarity of the pair potential has several consequences: (i) there is no specific anchoring between the LCs and the MNPs beyond anchoring among the LCs and the MNPs, (ii) polydispersity-induced phenomena are absent here, and (iii) unlike Refs. [12, 26, 31, 32, 43, 72], there is no structure formation due to the direct magnetic dipole-dipole interaction between the MNPs. These properties of the model enable us to isolate the role of the selectively controlled direction of particles and study its effect on the structure and rheology of the whole system.

We have implemented such a system in Ref. [34], where we focus on a temperature and density close to the isotropic state (in absence of the magnetic field and shear). The MNPs constitute 5\% of the system's particles with magnetic dipoles which are embedded along their longest axes. The simulations are performed using NEMD in NVT-ensemble (for the simulation details see Ref. [34]).

Similar to a rheometer, the liquid is confined between two walls (along the $z$-axis, see Figure 11) and it is sheared by a relative motion of these walls (along the $x$-direction). These rigid and impenetrable walls are formed at relatively high temperatures (deep in the isotropic state) by a sudden freezing of two slabs of particles. The thickness of the walls is chosen to be larger than the GB potential cutoff in our simulation setup. These frozen slabs serve as solid, impenetrable, and rough walls, with which the system is sheared until it reaches the steady-state.

After reaching the steady state, the magnetic field is increased gradually in time (in a linear fashion) from zero to a saturation value where all the MNPs fully align with the field. This induces, strictly speaking, a time-dependence into the protocol which is, however, computationally advantageous (as compared to extensive steady-state simulations). In Ref. [34], we examined the effect of the field on the system for three different field directions: (i) along $\mathbf{n}^{(0)}$, (ii) perpendicular to $\mathbf{n}^{(0)}$ in the $y z$-plane (vorticity direction), and (iii) perpendicular to $\mathbf{n}^{(0)}$ in the $x z$-plane (shear plane), where 


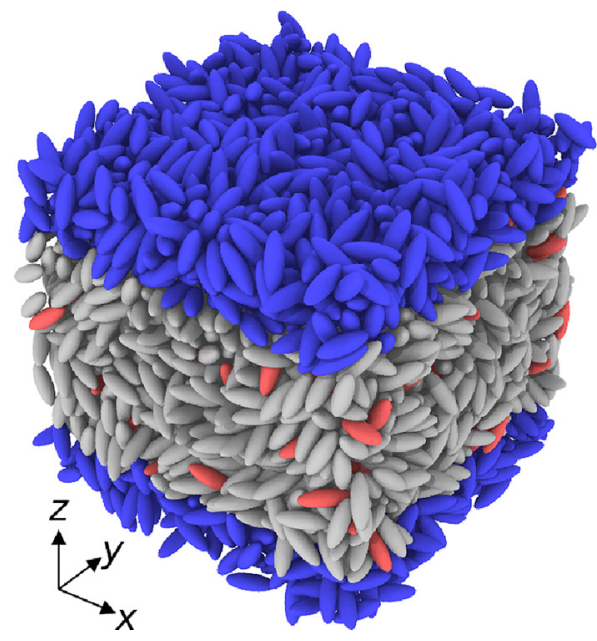

Figure 11: A snapshot of the simulation setup in absence of shear and magnetic field. The magnetic and non-magnetic particles are depicted by gray and red ellipsoids, respectively. The wall particles are distinguished by their blue color. The system, which is in an isotropic state, is confined by the walls along the $z$-axis and has periodic boundary conditions along $x$ - and $y$-directions. Reprinted from Ref. [34].

$\mathbf{n}^{(0)}$ represents the nematic director in absence of the field. The shear stress, $\sigma_{x z}$, is calculated via summation of the $x$-component of the forces exerted by the liquid particles on the upper or lower wall particles.

\subsubsection{Shear stress vs. magnetic field}

Investigating the shear stress (or equivalently the viscosity) as a function of the applied external field, one observes that the present system shows an unusual and intriguing behavior [34], see Figure 12. The most pronounced effect of the magnetic field occurs in case (iii), where we observe, remarkably, a non-monotonic behavior. In contrast, there are no marked field-induced changes of $\sigma_{x z}$ in the parallel case. We note that, due to our simulation protocol, the observed non-monotonicity is actually a time-dependent effect; yet we have checked that it occurs for a wide range of rates by which the field is changed.

In the subsequent discussion, we restrict ourselves to case (iii) and investigate the origins of the observed non-monotonicity as a function the magnetic field strength $H:=|\mathbf{H}|$. More specifically, we find the signatures of the aforementioned nonmonotonic behavior in the structure of the system. As shown by the snapshots in Figure 12, the magnetic field affects both the orientational and the positional order of the MNPs.

\subsubsection{Orientational and positional ordering}

A quantitative analysis of the nematic director of the MNPs $\left(\widehat{n}_{\text {MNP }}\right)$ reveals that there is a strong correlation between the magnetic field-induced deviation of $\hat{n}_{\mathrm{MNP}}$ from $\mathbf{n}^{(0)}$ and the increase in the shear stress (see Figure 13). Interestingly by increasing $H$ from zero, 


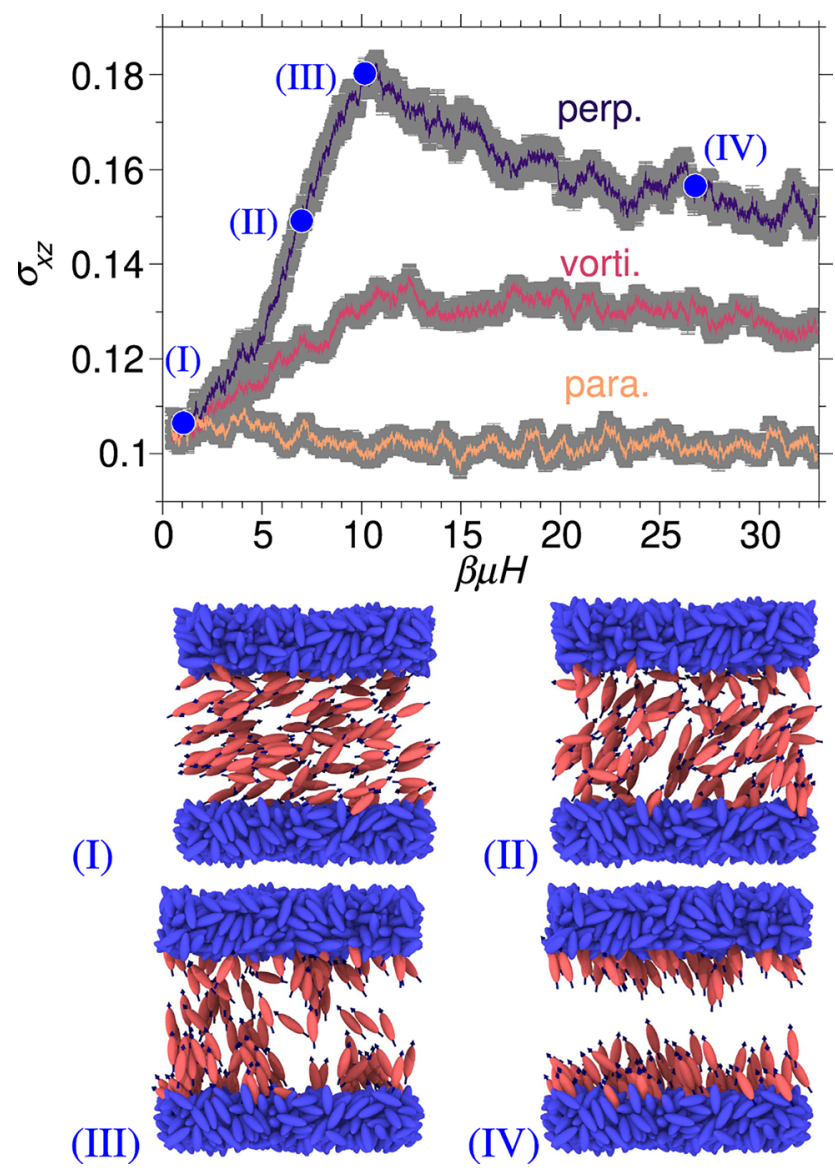

Figure 12: Upper panel: Shear stress as a function of the strength of the scaled magnetic field at three different directions: (i) the direction parallel to $\mathbf{n}^{(0)}$, (ii) the vorticity direction, which is parallel to the $y$-axis, and (iii) the direction perpendicular to $\mathbf{n}^{(0)}$ in the $x z$-plane. Here $H$ is the magnetic field strength, $\mu$ is the magnetic dipole moment, and $\beta=1 /\left(k_{B} T\right)$ is the inverse thermal energy. Lower panel: Representative configurations associated with the four values of the field, which are indicated by bullets and the Roman numbers on the curve. Figures in both panels are adapted from Ref. [34].

for small values of $H$, the orientations of the MNPs show a Fréederickz-like transition. For magnetic field strength larger than the threshold value for this transition, the MNPs start deviating from the shear-induced orientation, which leads to an increase in shear stress (see Figure 13). This correlation can be understood by considering that the misalignement of the MNPs restricts the shear-induced motion of the LCs, and therefore, increases the shear stress.

One should note that not only the orientational order of the MNPs, but also that of the LCs is affected by the magnetic field, although the LCs themselves are not susceptible to the magnetic field (see Figure 13). This is an indirect effect: The magnetic 
field re-orients the MNPs, which in turn, affects the orientation of the neighboring LCs due to the anisotropic steric interactions between the MNPs and LCs.

So far, we have focused on the correlation between the field-induced orientational ordering of the magnetic particles and the shear stress at small field strengths. Upon further increase of the magnetic field, a significant qualitative change in the spatial distribution of the MNPs occurs which correlates with the decrease of the shear stress. This qualitative change is illustrated by the four representative configurations at different values of $\mu \mathrm{H}$ in Figure 12: Starting from a relatively homogeneous distribution of MNPs at small $H$, further increase of $H$ leads to a spatial demixing between MNPs and LCs, where the MNPs assemble at the walls. One can quantify these structural transformations by measuring the averaged number density profile of MNPs as a function of the external field. The results are plotted in Figure 14. At small field strengths (state I and II) a uniform spatial distribution of the MNPs (and thus, also the LCs) is observed. In qualitative contrast to that, at large field strengths (state points III and IV) one observes a pronounced double-peak structure of the MNPs density profile, reflecting the assembly of the MNPs at the walls. Using a quantitative scalar measure for the degree of inhomogeneity of MNPs, one can show that the demixing occurs in the same range of field strength as the drop in the shear stress [34]. This correlation can be understood as follows: as soon as the system demixes, that is, at large values of $\mu H$, the MNPs are concentrated close to the walls, and this provides a channel for the LCs in

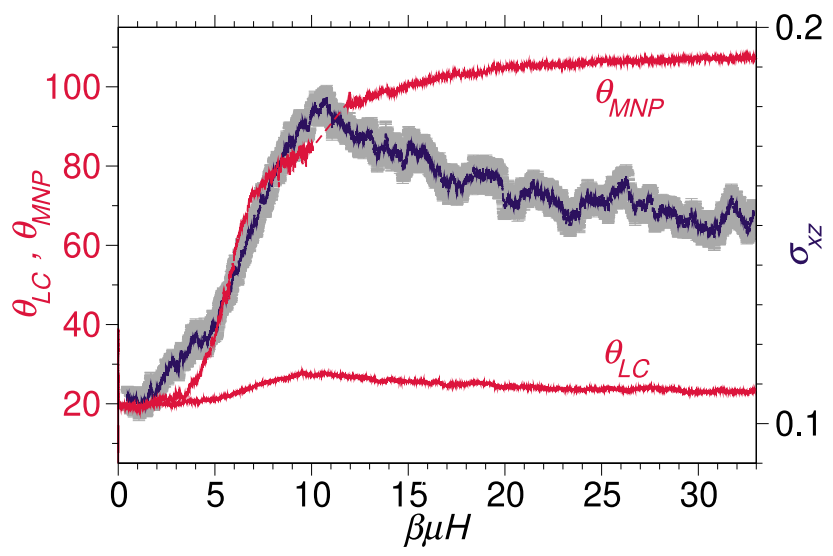

Figure 13: The angles between the nematic directors, i.e., $\widehat{n}_{\mathrm{LC}}$ and $\widehat{n}_{\mathrm{MNP}}$ ) with the shear direction ( $x$ direction) are shown on the left vertical axis. In absence of the magnetic field (i.e., $H=0$ ) the LCs and the MNPs are indistinguishable, and therefore the value of these angles coincide. The nematic director remains undistorted up to a threshold value of the magnetic field (here $\beta \mu H_{\mathrm{th}} \simeq 3.3$ ), and for $H>H_{\text {th }}$ it starts deviating from the $H=0$ direction. This deviation increases as the magnetic field increases and eventually all the MNPs are fully aligned with the direction of the magnetic field. This is Reminiscent of Fréderickz transition. The shear stress is shown on the right vertical axis as a function of $H$. Reprinted from Ref. [34]. 


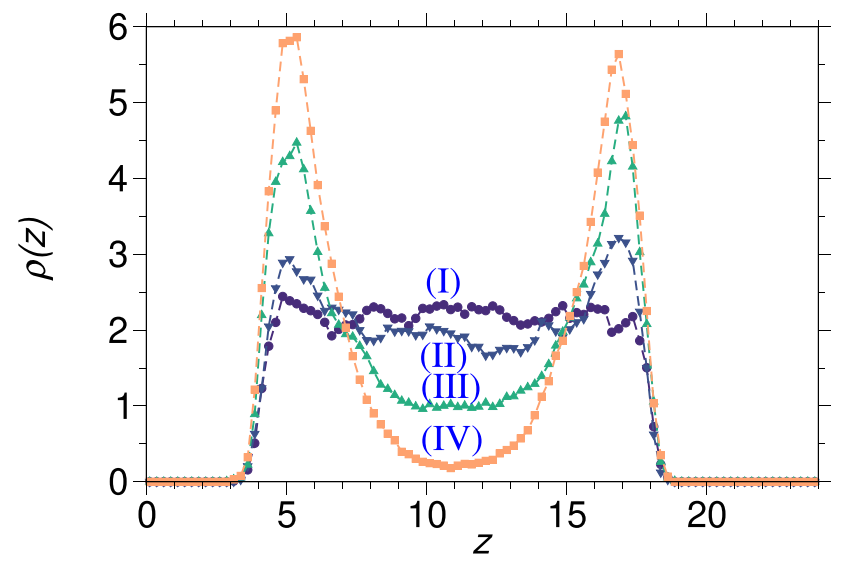

Figure 14: The density profiles of the MNPs at different magnetic field values, where $\rho\left(z_{0}\right):=n\left(z_{0}-\delta z / 2\right.$, $\left.z_{0}+\delta z / 2\right) /\left(L^{2} \delta z\right)$, where $n$ is the instantaneous number of MNPs between planes $z=z_{0}-\delta z / 2$ and $z=z_{0}+\delta z / 2$, and $\delta z=0.25$ is the discretization resolution along the $z$-axis. The roman numbers refer to the same numbers in Figure 12. For small fields, a relatively uniform spatial distribution is obtained, in contrast to strong fields where a double-peaked profile emerges. The figure is adapted from Ref. [34].

which they can flow without (orientational and positional) disturbances from the MNPs.

There remains the question as to why the system demixes at all. In Ref. [34] we have proposed to explain the demixing within a quasi-equilibrium picture, namely as a competition between mixing and the packing entropies. A large packing entropy is achieved when the particles of similar orientations are neighbors, whereas a higher mixing entropy is obtained via a uniform distribution of particles over the whole system. As long as the misalignment between the nematic director of the two species is small, the mixing entropy dominates and all the particles (including the MNPs) are distributed uniformly in the system. In contrast, at large misalignments (i.e. large fields), the system gains entropy by packing the particles of similar orientations together. Hence, demixing occurs.

\section{Outlook}

In this article, we have summarized recent computer simulation studies of soft composites of LCs and MNPs in equilibrium and under shear flow. The results indicate a surprisingly rich and complex behavior of such systems already under the simplified conditions assumed in our models. This indicates, together with many recent observations from the experimental side (see, e.g., $[11,13,14,58]$ ), that LC-MNP are ideal candidates for soft materials which are tunable by external fields, but also show 
self-assembly (by the involved interactions), and dynamical self-organization under driving forces such as shear.

There are several future directions of research which we would like to propose. First, the study of mixtures involving anisotropic MNPs with significant dipolar interactions (which we have neglected in Section 4.2) is only in its infancy. It is indeed well established that the shape of magnetic particles strongly affects the lowest-energy configurations of a pair of particles. For example, magnetic rods with longitudinal dipoles tend to form anti-parallel side-by-side configurations $[25,26]$ rather than the head-tail configurations familiar from magnetic spheres. This, in turn, determines the type of self-assembled clusters as well as the structures formed in a (constant) magnetic field. It seems therefore very promising to perform computational studies of interacting non-spherical MNPs of different size in LC matrices. One could also consider particles where the dipole is displaced from the center (similar to the studies for spherical MNPs [73-78]) or along the shortest axis, as done in recent experiments [79]. Depending on different dipole embeddings, we speculate to find intriguing structures which can be stabilized or destabilizes by an external magnetic field. The latter can also be timedependent, an example being a planar rotating field which is known to lead to new structure formation (such as sheets) already in pure dipolar systems [80, 81]. Further, given the observations discussed in the present article it is clear that all these structures will also have a profound impact (and vice versa) on the rheology. Finally, for a better comparison with real systems, it will be important to overcome the computational difficulties when studying mixtures which much smaller or larger MNPs (relative to the size of the LCs).

Acknowledgments: Financial support from the German Science Foundation (DFG) via priority programme SPP 1681 is gratefully acknowledge.

Author contribution: All the authors have accepted responsibility for the entire content of this submitted manuscript and approved submission.

Research funding: The study was financially supported from the German Science Foundation (DFG) via priority programme SPP 1681.

Conflict of interest statement: The authors declare no conflicts of interest regarding this article.

\section{References}

1. Brochard F, de Gennes P. Theory of magnetic suspensions in liquid crystals. J Phys 1970;31:691.

2. Odenbach S. Magnetic fluids - suspensions of magnetic dipoles and their magnetic control. J Phys Condens Matter 2003;15:S1497.

3. Wells J, Kazakova O, Posth O, Steinhoff U, Petronis S, Bogart LK, et al. Standardisation of magnetic nanoparticles in liquid suspension. J Phys Appl Phys 2017;50:383003.

4. Liebert L, Martinet A. Coupling between nematic lyomesophases and ferrofluids. J Phys Lett 1979; 40:363. 
5. Chen S-H, Amer NM. Observation of macroscopic collective behavior and new texture in magnetically doped liquid crystals. Phys Rev Lett 1983;51:2298.

6. Neto AF, Saba M. Determination of the minimum concentration of ferrofluid required to orient nematic liquid crystals. Phys Rev A 1986;34:3483.

7. Podoliak N, Buchnev O, Buluy O, D’Alessandro G, Kaczmarek M, Reznikov Y, et al. Macroscopic optical effects in low concentration ferronematics. Soft Matter 2011;7:4742.

8. Kopčanskỳ P, Tomašovičová $N$, Koneracká $M$, Závišová $V$, Timko $M$, Džarová A, et al. Structural changes in the $6 \mathrm{CHBT}$ liquid crystal doped with spherical, rodlike, and chainlike magnetic particles. Phys Rev E 2008;78:011702.

9. Buluy O, Nepijko S, Reshetnyak V, Ouskova E, Zadorozhnii V, Leonhardt A, et al. Magnetic sensitivity of a dispersion of aggregated ferromagnetic carbon nanotubes in liquid crystals. Soft Matter 2011;7:644.

10. Kredentser S, Buluy O, Davidson P, Dozov I, Malynych S, Reshetnyak V, et al. Strong orientational coupling in two-component suspensions of rod-like nanoparticles. Soft Matter 2013;9:5061.

11. May K, Stannarius R, Klein S, Eremin A. Electric-field-induced phase separation and homogenization dynamics in colloidal suspensions of dichroic rod-shaped pigment particles. Langmuir 2014;30:7070.

12. May K, Eremin A, Stannarius R, Peroukidis SD, Klapp SH, Klein S. Colloidal suspensions of rodlike nanocrystals and magnetic spheres under an external magnetic stimulus: experiment and molecular dynamics simulation. Langmuir 2016;32:5085.

13. Mertelj A, Lisjak D, Drofenik M, Čopič M. Ferromagnetism in suspensions of magnetic platelets in liquid crystal. Nature 2013;504:237.

14. Mertelj A, Osterman N, Lisjak D, Čopič M. Magneto-optic and converse magnetoelectric effects in a ferromagnetic liquid crystal. Soft Matter 2014;10:9065.

15. Kyrylyuk AV, Hermant MC, Schilling T, Klumperman B, Koning CE, Van der Schoot P. Controlling electrical percolation in multicomponent carbon nanotube dispersions. Nat Nanotechnol 2011;6: 364.

16. Podoliak N, Buchnev O, Bavykin DV, Kulak AN, Kaczmarek M, Sluckin TJ. Magnetite nanorod thermotropic liquid crystal colloids: synthesis, optics and theory. J Colloid Interf Sci 2012;386:158.

17. Martinez-Pedrero F, Cebers A, Tierno P. Dipolar rings of microscopic ellipsoids: magnetic manipulation and cell entrapment. Phys Rev Appl 2016;6:034002.

18. Kredentser S, Kulyk M, Kalita V, Slyusarenko K, Reshetnyak VY, Reznikov YA. Magneto-induced anisotropy in magnetic colloids of superparamagnetic magnetite nanoparticles in an external magnetic field. Soft Matter 2017;13:4080.

19. Poulin P, Stark H, Lubensky T, Weitz D. Novel colloidal interactions in anisotropic fluids. Science 1997;275:1770.

20. Poulin P, Weitz D. Inverted and multiple nematic emulsions. Phys Rev E 1998;57:626.

21. Meeker S, Poon W, Crain J, Terentjev E. Colloid-liquid-crystal composites: an unusual soft solid. Phys Rev E 2000;61:R6083.

22. Nazarenko V, Nych A, Lev B. Crystal structure in nematic emulsion. Phys Rev Lett 2001;87:075504.

23. Lapointe CP, Mason TG, Smalyukh II. Shape-controlled colloidal interactions in nematic liquid crystals. Science 2009;326:1083.

24. Evans JS, Beier CN, Smalyukh II. Alignment of high-aspect ratio colloidal gold nanoplatelets in nematic liquid crystals. J Appl Phys 2011;110:033535.

25. McGrother SC, Gil-Villegas A, Jackson G. The effect of dipolar interactions on the liquid crystalline phase transitions of hard spherocylinders with central longitudinal dipoles. Mol Phys 1998;95: 657.

26. Alvarez CE, Klapp SH. Percolation and orientational ordering in systems of magnetic nanorods. Soft Matter 2012;8:7480. 
27. Zadorozhnii V, Vasilev A, Reshetnyak VY, Thomas K, Sluckin T. Nematic director response in ferronematic cells. Europhys Lett 2005;73:408.

28. Raikher YL, Stepanov VI, Zakhlevnykh AN. Mean-field description of the order-disorder phase transition in ferronematics. Soft Matter 2013;9:177.

29. Peroukidis SD, Klapp SH. Spontaneous ordering of magnetic particles in liquid crystals: from chains to biaxial lamellae. Phys Rev E 2015;92:010501.

30. Peroukidis SD, Lichtner K, Klapp SH. Tunable structures of mixtures of magnetic particles in liquidcrystalline matrices. Soft Matter 2015;11:5999.

31. Peroukidis SD, Klapp SH. Orientational order and translational dynamics of magnetic particle assemblies in liquid crystals. Soft Matter 2016;12:6841.

32. Shrivastav GP, Klapp SH. Anomalous transport of magnetic colloids in a liquid crystal-magnetic colloid mixture. Soft Matter 2019;15:973.

33. Shrivastav GP, Siboni NH, Klapp SHL. Steady-state rheology and structure of soft hybrid mixtures of liquid crystals and magnetic nanoparticles. Soft Matter 2020;16:2516.

34. Siboni NH, Shrivastav GP, Klapp SH. J Chem Phys 2020;152:024505.

35. Odenbach S. Recent progress in magnetic fluid research. J Phys Condens Matter 2004;16:R1135.

36. Torres-Díaz I, Rinaldi C. Recent progress in ferrofluids research: novel applications of magnetically controllable and tunable fluids. Soft Matter 2014;10:8584.

37. Felicia LJ, Vinod S, Philip J. Recent advances in magnetorheology of ferrofluids (magnetic nanofluids)-a critical review. J Nanofluids 2016;5:1.

38. Yannopapas V, Klapp SH, Peroukidis SD. Magneto-optical properties of liquid-crystalline ferrofluids. Opt Mater Express 2016;6:2681.

39. De Miguel E, Rull LF, Chalam MK, Gubbins KE. Liquid crystal phase diagram of the Gay-Berne fluid. Mol Phys 1991;74:405.

40. Schoen M, Klapp S. Rev Comp Chem 2007;24:1.

41. Klokkenburg M, Erné BH, Meeldijk JD, Wiedenmann A, Petukhov AV, Dullens RP, et al. In Situlmaging of field-induced hexagonal columns in magnetite ferrofluids. Phys Rev Lett 2006;97: 185702.

42. Gerth-Noritzsch M, Borin DY, Odenbach S. Anisotropy of the magnetoviscous effect in ferrofluids containing nanoparticles exhibiting magnetic dipole interaction. J Phys Condens Matter 2011;23: 346002.

43. Sreekumari A, Ilg P. Slow relaxation in structure-forming ferrofluids. Phys Rev E 2013;88:042315.

44. Rovigatti L, Russo J, Sciortino F. Structural properties of the dipolar hard-sphere fluid at low temperatures and densities. Soft Matter 2012;8:6310.

45. De Gennes P-G, Prost J. The physics of liquid crystals, 83 Oxford: Clarendon Press; 1993.

46. Peroukidis SD, Vanakaras AG. Phase diagram of hard board-like colloids from computer simulations. Soft Matter 2013;9:7419.

47. Cuetos A, Galindo A, Jackson G. Thermotropic biaxial liquid crystalline phases in a mixture of attractive uniaxial rod and disk particles. Phys Rev Lett 2008;101:237802.

48. Jordanovic J, Jäger S, Klapp SH. Crossover from normal to anomalous diffusion in systems of fieldaligned dipolar particles. Phys Rev Lett 2011;106:038301.

49. Jaeger S, Stark H, Klapp SH. Dynamics of cluster formation in driven magnetic colloids dispersed on a monolayer. J Phys Condens Matter 2013;25:195104.

50. de Miguel E, Rull LF, Gubbins KE. Dynamics of the Gay-Berne fluid. Phys Rev A 1992;45:3813.

51. Allen MP. Diffusion coefficient increases with density in hard ellipsoid liquid crystals. Phys Rev Lett 1990;65:2881.

52. Bonn D, Denn MM, Berthier L, Divoux T, Manneville S. Yield stress materials in soft condensed matter. Rev Mod Phys 2017;89:035005. 
53. Germano G, Schmid F. Nematic-isotropic interfaces under shear: A molecular-dynamics simulation. J Chem Phys 2005;123:214703.

54. Ripoll M, Holmqvist P, Winkler R, Gompper G, Dhont J, Lettinga M. Attractive colloidal rods in shear flow. Phys Rev Lett 2008;101:168302.

55. Ripoll M, Winkler R, Mussawisade K, Gompper G. Mesoscale hydrodynamics simulations of attractive rod-like colloids in shear flow. J Phys Condens Matter 2008;20:404209.

56. Sreekumari A, Ilg P. Anisotropy of magnetoviscous effect in structure-forming ferrofluids. Phys Rev E 2015;92:012306.

57. Sebastián N, Osterman N, Lisjak D, Čopič M, Mertelj A. Director reorientation dynamics of ferromagnetic nematic liquid crystals. Soft Matter 2018;14:7180.

58. Potisk T, Mertelj A, Sebastián N, Osterman N, Lisjak D, Brand HR, et al. Magneto-optic dynamics in a ferromagnetic nematic liquid crystal. Phys Rev E 2018;97:012701.

59. Zarubin G, Bier M, Dietrich S. A ferronematic slab in external magnetic fields. Soft Matter 2018;14: 9806.

60. Zarubin G, Bier M, Dietrich S. Effective landau theory of ferronematics. J Chem Phys 2018;149: 054505.

61. Kikuchi N, Horbach J. Mobile particles in an immobile environment: Molecular dynamics simulation of a binary Yukawa mixture. Europhys Lett 2007;77:26001.

62. Zaccarelli E, Liddle SM, Poon WC. On polydispersity and the hard sphere glass transition. Soft Matter 2015;11:324.

63. Heckendorf D, Mutch K, Egelhaaf S, Laurati M. Size-dependent localization in polydisperse colloidal glasses. Phys Rev Lett 2017;119:048003.

64. Ferreiro-Córdova C, Wensink H. Spinodal instabilities in polydisperse lyotropic nematics. J Chem Phys 2016;145:244904.

65. Verhoeff A, Wensink H, Vis M, Jackson G, Lekkerkerker H. Liquid crystal phase transitions in systems of colloidal platelets with bimodal shape distribution. J Phys Chem B 2009;113:13476.

66. Speranza A, Sollich P. Simplified onsager theory for isotropic-nematic phase equilibria of length polydisperse hard rods. J Chem Phys 2002;117:5421.

67. Blaak R, Auer S, Frenkel D, Löwen H. Homogeneous nucleation of colloidal melts under the influence of shearing fields. J Phys Condens Matter 2004;16:S3873.

68. Mokshin AV, Barrat J-L. Shear-induced crystallization of an amorphous system. Phys Rev E 2008; 77:021505.

69. Mandal S, Gross M, Raabe D, Varnik F. Heterogeneous shear in hard sphere glasses. Phys Rev Lett 2012;108:098301.

70. Shrivastav GP, Chaudhuri P, Horbach J. Heterogeneous dynamics during yielding of glasses: effect of aging. J Rheol 2016;60:835.

71. Hess S, Kröger M. Regular and chaotic orientational and rheological behaviour of liquid crystals. J Phys Condens Matter 2004;16:S3835.

72. Schmidle H, Hall CK, Velev OD, Klapp SH. Phase diagram of two-dimensional systems of dipole-like colloids. Soft Matter 2012;8:1521.

73. Kantorovich S, Weeber R, Cerda JJ, Holm C. Ferrofluids with shifted dipoles: ground state structures. Soft Matter 2011;7:5217.

74. Klinkigt M, Weeber R, Kantorgovich S, Holm C. Cluster formation in systems of shifted-dipole particles. Soft Matter 2013;9:3535.

75. Morphew D, Chakrabarti D. Supracolloidal reconfigurable polyhedra via hierarchical selfassembly. Soft Matter 2016;12:9633.

76. Steinbach G, Gemming S, Erbe A. Non-equilibrium dynamics of magnetically anisotropic particles under oscillating fields. Eur Phys J E 2016;39:69. 
77. Yener AB, Klapp SH. Self-assembly of three-dimensional ensembles of magnetic particles with laterally shifted dipoles. Soft Matter 2016;12:2066.

78. Rutkowski DM, Velev OD, Klapp SH, Hall CK. Simulation study on the structural properties of colloidal particles with offset dipoles. Soft Matter 2017;13:3134.

79. Martinez-Pedrero F, Cebers A, Tierno P. Orientational dynamics of colloidal ribbons selfassembled from microscopic magnetic ellipsoids. Soft Matter 2016;12:3688.

80. Jäger S, Klapp SH. Pattern formation of dipolar colloids in rotating fields: layering and synchronization. Soft Matter 2011;7:6606.

81. Yan J, Bae SC, Granick S. Rotating crystals of magnetic Janus colloids. Soft Matter 2015;11:147. 